Innovación y Desarrollo Tecnológico y Social (2020) 2 (2): 237-251- Número especial COVID-19

\title{
Los pueblos indígenas son parte de la solución
}

\author{
Alternativas comunitarias de cuidado frente a la crisis sanitaria
}

Carolina Maidana "; Alejandro Martínez 2; Liliana Tamagno 2; Stella Maris García 2; Diego Bermeo 3; Lucía Aljanati 2; Laura Aragon 2; Nadia Voscoboinik 2; Sofía Silva 2; Fernanda Alonso 2; Paloma Romero Gozzi ${ }^{4}$; Facundo Escobar ${ }^{5}$; Juan Manuel Di Socio 6 ${ }^{1}$ CONICET/LIAS-FCNyM-UNLP - lias@fcnym.unIp.edu.ar, ${ }^{2}$ LIAS-FCNyM-UNLP; ${ }^{3}$ Facultad de Trabajo Social-UNLP; ${ }^{4}$ FCNyM-UNLP; ${ }^{5}$ Facultad de Periodismo y Comunicación Social-UNLP; ${ }^{6}$ Universidad Nacional Arturo Jauretche

Resumen: La pandemia de coronavirus pone de manifiesto el modelo de sociedad que comenzó a imponerse a nivel mundial a partir del siglo XVII (de Souza Santos 2010). Una de las características esenciales de este modelo es la apropiación violenta y devastadora de la naturaleza, de los "bienes comunes", de "nuestro hogar común", de "nuestra madre tierra", en términos de los pueblos indígenas, cuyos sistemas de valores/cosmovisiones/ontologías orientan las relaciones hacia la alteridad humana y no humana en términos de reciprocidad/don/protección y no de producción y apropiación depredatoria. De allí que aparezca la expresión "Ios pueblos indígenas son parte de la solución" para dar cuenta de que sus prácticas y conocimientos colectivos-comunitarios constituyen alternativas de vida que pueden aportar a la reducción de fenómenos como el calentamiento global, la pérdida de biodiversidad, la ocurrencia cada vez más frecuente de eventos climáticos extremos y el brote de epidemias y pandemias como la ocasionada por el virus SARS-CoV-2. Esta situación, que conmociona al mundo entero, nos convoca a aunar esfuerzos para enfrentar los nuevos desafíos que plantea y los viejos problemas que agudiza.

En el caso de los pueblos indígenas de Argentina, los efectos de la pandemia han exacerbado las complejas situaciones que estos colectivos venían enfrentando en torno a la salud, la educación, el ambiente, la alimentación, el trabajo, el acceso a la información, la judicialización, la criminalización y el racismo. Esto plantea la necesidad de profundizar en el conocimiento de las formas en que se han agravado desigualdades preexistentes, a los fines de proponer medidas 
Innovación y Desarrollo Tecnológico y Social (2020) 2 (2): 237-251- Número especial COVID-19

concretas para su atención. A su vez, las comunidades indígenas están cumpliendo con mucho esfuerzo la medida de Aislamiento Social Preventivo y Obligatorio (ASPO) establecida por Decreto 297/2020, movilizando y desplegando saberes y prácticas colectivas-comunitarias ante la preocupación por adecuar su dinámica sociocultural a los requerimientos de este decreto. Elaboramos entonces el proyecto "Saberes y prácticas indígenas en un contexto de emergencia sanitaria: aportes a la salud, la educación y el ambiente en el marco de la planificación de estrategias de control, prevención y monitoreo del covid-19", a los fines de profundizar en el conocimiento y evaluación del impacto del ASPO en los pueblos indígenas, pero también y fundamentalmente, para reconocer y registrar conocimientos y prácticas que -fundadas en el don y la reciprocidad- pueden constituir, en este estado de las cosas, un aporte a la resolución de problemáticas emergentes y al diseño e implementación de programas sociales que los involucren. Para ello hemos propuesto una metodología que prioriza el respeto y el compromiso con los sujetos junto a los cuales construimos conocimiento $y_{1}$ dados los sabidos condicionamientos que restringen hasta hoy la circulación y reunión de personas en todo el territorio nacional, la puesta en marcha de técnicas propias de una "etnografía digital" que nos permitirá alcanzar los objetivos propuestos respetando las medidas preventivas: observación y entrevistas "a distancia" mediadas por distintas tecnologías (plataformas de video conferencia, servicios de telefonía y mensajería instantánea, correos electrónicos, redes sociales, entre otras.). Los pueblos indígenas deben formar parte de la planificación de estrategias de control, prevención y monitoreo del COVID-19. No se puede y no se debe subestimar el valor de sus prácticas y conocimientos, que revisten una singular importancia en esta coyuntura de emergencia sanitaria, al representar otras posibles formas de "cuidarse entre todos". Y, en este sentido, son esenciales también para encontrar soluciones a futuro, es decir, para pensar otras emergencias en la pospandemia.

Palabras clave: pueblos indígenas; pandemia; cuidados; diálogo de saberes

Recibido: 01/10/2020 Aceptado: 08/10/2020

DOI: https://doi.org/10.24215/26838559e024 
Innovación y Desarrollo Tecnológico y Social (2020) 2 (2): 237-251- Número especial COVID-19

\section{Indigenous peoples are part of the solution}

Community care alternatives in the face of the health crisis

Abstract: The coronavirus pandemic illustrates the model of society that began to be imposed globally since the 17th century (de Souza Santos 2010). One of the essential characteristics of this model is the violent and devastating appropriation of nature, of the "common goods" of "our common home", of "our mother earth", in terms of indigenous peoples, whose value systems/cosmovision's/ontologies orient relations toward human and non-human otherness in terms of reciprocity/gift/protection rather than production and predatory appropriation. This is why the expression "indigenous peoples are part of the solution" appears, to show that their collective-community practices and knowledge constitute alternative ways of life that can contribute to the reduction of phenomena such as global warming, loss of biodiversity, the increasingly frequent occurrence of extreme climate events, and the outbreak of epidemics and pandemics such as that caused by the SARS-CoV-2 virus. This situation, which shocks the entire world, calls on us to join forces to face the new challenges it poses and the old problems it exacerbates.

In the case of the indigenous peoples of Argentina, the effects of the pandemic have exacerbated the complex situations that these groups have been facing in the areas of health, education, environment, food, work, access to information, judicialization, criminalization, and racism. This raises the need to deepen the knowledge of the ways in which pre-existing inequalities have been aggravated, in order to propose concrete measures for their attention. At the same time, indigenous communities are making a great effort to comply with the measure of Preventive and Obligatory Social Isolation (ASPO, Spanish initials) established by Decree 297/2020, mobilizing and deploying collective-community knowledge and practices in order to adapt their sociocultural dynamics to the requirements of this decree. We developed the project "Indigenous Knowledge and Practices in a Health Emergency Context: Contributions to Health, Education and the Environment in the Framework of Planning Strategies for the Control, Prevention and Monitoring of Covid-19", with the aim of deepening knowledge and evaluating the impact of ASPO on indigenous peoples, but also and fundamentally, to recognize and record knowledge and practices that - based on gift and reciprocity - can constitute, in this state of things, a contribution to the resolution of emerging problems and the design and implementation of 
Innovación y Desarrollo Tecnológico y Social (2020) 2 (2): 237-251- Número especial COVID-19

social programs that involve them. To this end, we have proposed a methodology that prioritizes respect and commitment to the subjects with whom we build knowledge and, given the known conditions that so far restrict the movement and gathering of people throughout the national territory, the implementation of techniques characteristic of a "digital ethnography" that will allow us to achieve the proposed objectives while respecting preventive measures: observation and interviews "at a distance" mediated by various technologies (video conference platforms, telephone and instant messaging services, emails, social networks, among others.)

Indigenous peoples must be part of the planning of strategies for control, prevention and monitoring of COVID-19. The value of their practices and knowledge cannot and should not be underestimated, as they are of singular importance in this health emergency situation, representing other possible ways of "taking care of each other". In this sense, they are also essential for finding future solutions, that is, for thinking about other post-pandemic emergencies.

Keywords: indigenous peoples; pandemic; care; dialogue of knowledge

\section{Novedad u originalidad local en el conocimiento}

En Argentina, el último Censo Nacional de Población, Hogares y Viviendas (2010) registró casi un millón (955.032) de personas que se reconocen como indígenas. El 82\% de este total (782.171) se asienta en zonas urbanas. En la Región Metropolitana de Buenos Aires (RMBA) aproximadamente 248.516 personas pertenecen a alguno de los 40 pueblos indígenas actualmente reconocidos en el país y 46 comunidades con personería jurídica registrada o en proceso de estarlo. Si bien estos números representan subregistros -originados en diversos criterios de identificación, lógicas clasificatorias y modos de implementación de las herramientas censales- dan cuenta de presencias históricamente negadas e 
Innovación y Desarrollo Tecnológico y Social (2020) 2 (2): 237-251- Número especial COVID-19

invisibilizadas. Ante la situación de pandemia se renueva la necesidad de comprender que el diseño de toda política pública debe partir inexorablemente del reconocimiento de las trayectorias de los pueblos indígenas y su situación actual. Por ello este planteo surge desde distintos campos disciplinares y proyectando una articulación con el Estado, para profundizar en el conocimiento de cómo transitan esta peculiar coyuntura los pueblos indígenas en la RMBA, de qué forma la emergencia sanitaria y las medidas de aislamiento preventivo y obligatorio impactan sobre su cotidianeidad social, laboral y educativa, y qué saberes colectivos comunitarios se ponen en juego para responder a la situación actual. El trabajo se desarrollará junto a tres comunidades pertenecientes a los Pueblos Indígenas Qom, Ava guaraní y Tonokote, las dos primeras están ubicadas en la periferia de la ciudad de La Plata y la última en el municipio de Florencio Varela.

\section{Grado de relevancia}

La relevancia del proyecto está dada, por un lado por la necesidad de responder a las difíciles situaciones que atraviesan los pueblos indígenas: se ha paralizado el empleo informal (una de las principales fuente de ingresos de las comunidades); han incrementado las situaciones de racismo; se han profundizado las dificultades que en muchas zonas existen para obtener agua potable, tener recolección de residuos, acceder a la atención médica y medicamentos; se han puesto de 
Innovación y Desarrollo Tecnológico y Social (2020) 2 (2): 237-251 - Número especial COVID-19

manifiesto las condiciones de hacinamiento en las que viven muchas comunidades, se observan impedimentos para acceder a la entrega de alimentos en comedores y/o escuelas (muchos cerraron o no alcanzan a cubrir las necesidades barriales/comunitarias en el nuevo contexto); la continuidad pedagógica se ve limitada por los problemas en la disponibilidad de dispositivos digitales y conectividad a internet y las comunidades vienen padeciendo dificultades para contar con insumos como tapabocas, lavandina y alcohol. Por otro, la relevancia radica en la posibilidad de pensar, a partir del diálogo de saberes, alternativas de control, prevención y monitoreo del COVID-19.

\section{Grado de pertinencia}

El cuidado de la salud es un proceso socialmente situado, en tanto las prácticas y las representaciones que pone en juego no se producen en un vacío histórico ni político, ni son llevadas a cabo por una sociedad aislada y homogénea (Colangelo, 2009). Así este proyecto se orienta a aportar elementos para el diseño y desarrollo de programas y planes sanitarios de carácter intercultural, entendiendo la interculturalidad en su sentido crítico. No sólo reconociendo la riqueza de las diferentes prácticas y representaciones sino también el hecho de que las relaciones establecidas no serán de carácter equitativo en tanto se den en el marco de una sociedad profundamente desigual y racista. El racismo -que es el modo de relación establecido por el capitalismo a fin de justificar la expropiación y 
Innovación y Desarrollo Tecnológico y Social (2020) 2 (2): 237-251- Número especial COVID-19

explotación (Menéndez, 1972)- se expresa no sólo cuando los pueblos indígenas se ven cotidianamente negados, explotados, sujetos a clientelismos de todo tipo y/o privados de sus derechos, sino también cuando su sobrevivencia depende de acciones que no revierten la inequidad y se los reduce a su sola condición de víctimas sin reconocer sus saberes y formas de organización fundadas en experiencias transmitidas de generación en generación. La propuesta de identificar los conocimientos y las prácticas colectivas-comunitarias que desplegadas para enfrentar la emergencia sanitaria se orienta a proponer un diálogo de saberes que, en este contexto de emergencia sanitaria, permita la construcción de soberanía desde los cimientos mismos de la sociedad, siendo los pueblos indígenas parte fundamental de esos cimientos (Tamagno 2020).

\section{Grado de demanda}

La Unidad Coronavirus -integrada por el Ministerio de Ciencia, Tecnología e Innovación (MINCyT), el Consejo Nacional de Investigaciones Científicas y Técnicas (CONICET) y la Agencia Nacional de Promoción de la Investigación, el Desarrollo Tecnológico y la Innovación (Agencia I+D+i)- se conformó frente a la crisis sanitaria con el objetivo de poner a disposición las capacidades de desarrollo de proyectos tecnológicos, recursos humanos, infraestructura y equipamiento que puedan ser requeridas para el diagnóstico e investigación sobre COVID-19. En marzo de 2020 la Comisión Ciencias Sociales de esta Unidad convocó a relevar el impacto social 
Innovación y Desarrollo Tecnológico y Social (2020) 2 (2): 237-251- Número especial COVID-19

de las medidas del ASPO. En este marco contactamos para una encuesta a referentes indígenas con vasto conocimiento sobre la situación de sus pueblos y comunidades. Tras este relevamiento, junto a otros equipos de investigación, en el mes de abril presentamos un informe titulado "Los efectos socioeconómicos y culturales de la pandemia COVID-19 y del aislamiento social, preventivo y obligatorio en las comunidades indígenas de la RMBA, NOA, NEA y Patagonia" y luego, sumando equipos de investigación de todo el país, presentamos el "Informe ampliado: efectos socioeconómicos y culturales de la pandemia COVID-19 y del aislamiento social, preventivo y obligatorio en los Pueblos Indígenas en Argentina Segunda etapa, junio 2020-“ donde relevamos la situación de 30 pueblos indígenas, advirtiendo la realidad que atraviesan estos colectivos en Argentina y sus reclamos ante derechos vulnerados. Pero, además de las denuncias, los propios referentes indígenas nos convocaron a valorar sus formas organizativas y sus conocimientos, por eso elaboramos este proyecto.

\section{Desarrollo del producto}

Nuestra experiencia con los pueblos indígenas nos dice que el vínculo con la universidad los fortalece al permitir que la valoración de lo propio sea percibida desde el afuera y por una institución que reconocen como prestigiosa. Por ello partimos de las recomendaciones presentadas en el “Protocolo de actuación para organismos gubernamentales de la provincia de Buenos Aires que reciben 
Innovación y Desarrollo Tecnológico y Social (2020) 2 (2): 237-251- Número especial COVID-19

demandas de personas, comunidades y pueblos indígenas por la efectivización de sus derechos" (2017), para conocer, comprender e indagar este nuevo contexto que genera la pandemia. Dicho documento fue diseñado en el marco de un protocolo adicional al Convenio de Cooperación Técnica y Asistencia Recíproca celebrado entre el defensor del pueblo de la Provincia de Buenos Aires y la Universidad Nacional de La Plata (UNLP)-Facultad de Ciencias Naturales y Museo (FCNyM), que forma parte de las políticas de defensa y promoción de los derechos humanos que la Defensoría del Pueblo de la provincia de Buenos Aires lleva adelante. Dichas recomendaciones son el resultado de una construcción colectiva, de una producción de conocimiento conjunto (Tamagno et al., 2005 - Maidana et. al., 2011) en el marco de una investigación de larga duración desarrollada desde el Laboratorio de Investigaciones en Antropología Social (LIAS) en diálogo con referentes y organizaciones indígenas y con el conocimiento antropológico producido en América Latina sobre los pueblos a los que estos referentes y organizaciones pertenecen:

1. Sobre la diversidad cultural y la autoadscripción. Señalamos que el diseño de toda política indígena debe partir de revisar la conceptualización de la Argentina como país "blanco" y sin "indios" que impide reconocer en toda su dimensión las trayectorias de los pueblos indígenas y su situación actual. En este contexto, se recuperan cifras censales y se indica la centralidad del principio de autoadscripción señalando que no corresponde a los funcionarios de organismos 
Innovación y Desarrollo Tecnológico y Social (2020) 2 (2): 237-251 - Número especial COVID-19

gubernamentales ni a los especialistas esgrimirse como "censores de indianidad" (Tamagno, 1991), sino que es facultad de las personas y las comunidades definirse como parte de estos pueblos, tal como lo establece el Convenio 169 de la Organización Internacional del Trabajo (OIT).

2. Sobre la libre determinación. Se indica -con base en la Declaración de las Naciones Unidas sobre los Derechos de los Pueblos Indígenas- la necesidad de reconocer todas las formas organizativas que se den a sí mismos estos pueblos y, en ese sentido, que el Estado-nación se identifique como plurinacional.

3. Sobre los liderazgos indígenas. Se plantea que los líderes deben ser considerados referentes en cuanto expresan, a nivel individual, las transformaciones, las luchas y las demandas de los colectivos con los cuales se referencian.

4. Sobre la consulta y el consentimiento libre, previo e informado. Se recuerda que es obligación de los Estados consultar a los pueblos indígenas sobre asuntos que los afecten en distintos contextos.

5. Sobre el acceso pleno al sistema de justicia del Estado nacional. Se indica que en todo procedimiento judicial se deben considerar las visiones de mundo de estos pueblos.

6. Sobre las transformaciones y las continuidades en las presencias indígenas. Se señala que al abordar la cuestión territorial es imprescindible considerar el 
Innovación y Desarrollo Tecnológico y Social (2020) 2 (2): 237-251- Número especial COVID-19

etnocidio y, al mismo tiempo, el hecho de que las culturas no mueren, sino que se transforman, y que estos procesos implican también movilidad geográfica.

7. Sobre su carácter de sujetos sociales. Se señala que es imprescindible preguntarse sobre quiénes produjeron las acciones que derivaron en las situaciones en que hoy se encuentran los pueblos indígenas, dejando en claro que las mismas son producto de la explotación y la desigualdad y no de condiciones internas de dichos conjuntos.

8. Sobre las lógicas comunitarias. Se indica que lo comunitario no se limita a la territorialidad y tampoco implica necesariamente hablar una lengua particular o compartir una religión: es sentirse parte de un conjunto, por lo cual sus derechos deben ser comprendidos en su carácter de derechos colectivos, a pesar de que la "lógica jurídica", fundada en una concepción individualista, dificulta la efectivización de estos "derechos colectivos", que solo tienen cabida en el marco de la persona o personería jurídica.

9. Sobre el concepto de pueblos indígenas. Se explica que el sujeto de derecho es el pueblo indígena y que el reconocimiento de las personerías jurídicas a sus comunidades es consecuencia de esta afirmación.

10. Sobre la historización de las demandas /tensiones / resistencias. Se indica que los acontecimientos que puntualmente generan demandas deben ser comprendidos en la dimensión histórica que los explica. 
Innovación y Desarrollo Tecnológico y Social (2020) 2 (2): 237-251- Número especial COVID-19

11. Sobre el racismo presente en el sentido común. Se señala que no debemos perder de vista que el racismo aparece ante los intereses de los sectores dominantes y sus proyectos en el contexto de la sociedad de mercado.

12. Sobre las políticas indígenas. Se concluye que las políticas culturales no deben desvincularse de las demandas sobre la tierra, la salud, la educación, el trabajo y la vivienda.

Respecto a este último punto -que llama la atención respecto de las políticas indígenas que con frecuencia apelan sólo a lo cultural relegando las luchas contra la exclusión, la inequidad socioeconómica y el racismo- se han manifestado un sinnúmero de veces los referentes indígenas con quienes dialogamos señalando que la búsqueda de soluciones requiere ir más allá del marco de referencia eurocéntrico, requiere del reconocimiento de la pluralidad de conocimientos; conocimientos plurales que, como señala de Souza Santos (2020), surgen y se hacen visibles a través de los actos de resistencia de los grupos sociales sometidos a la injusticia, opresión o destrucción sistemática en manos del capitalismo. “Las alternativas entrarán, cada vez con más frecuencia, en la vida de los ciudadanos a través de la puerta trasera de crisis pandémicas, desastres ambientales y colapsos financieros" (de Souza Santos 2020:22).

En el contexto de las consideraciones señaladas cabe alertar que esta producción de conocimiento ya sistematizada operará como basamento y pautas de acción para avanzar con el proyecto indicado. Actualmente, estamos trabajando 
Innovación y Desarrollo Tecnológico y Social (2020) 2 (2): 237-251- Número especial COVID-19

problemáticas en torno al acceso y manejo de las tecnologías de la información y comunicación (TICS), para garantizar la conectividad y la disponibilidad de dispositivos. En el contexto de aislamiento el no acceso a las TICs profundiza las desigualdades. Algo que se ha reconocido recientemente a través del DNU 690/20, mediante el cual el Poder Ejecutivo nacional determinó que los Servicios de las Tecnologías de la Información y las Comunicaciones (TIC) serán considerados servicios públicos esenciales y estratégicos. También, en esta primera etapa se está confeccionando la guía de preguntas de un cuestionario semiestructurado que tiene por objetivo relevar las condiciones de acceso a distintos servicios por parte de las comunidades involucradas e indagar sobre el conocimiento, el acceso a la información y los cuidados llevados adelante en las comunidades en relación al COVID-19. La misma será implementada vía telefónica a los referentes de las tres comunidades con las que trabajamos. La información recopilada se organizará en torno a ejes analíticos, tales como: a) saberes colectivos comunitarios que se ponen en juego para responder a la situación actual y, paralelamente, evaluar la posibilidad de planificar acciones a futuro, ensayando la factibilidad de que actúen como agentes multiplicadores de las experiencias y como gestores de nuevas iniciativas en otras comunidades; b) estrategias para atender problemáticas vinculadas a la subsistencia, la salud, la comunicación, el ambiente, la educación y que aporten a la afirmación identitaria; y c) soluciones 
Innovación y Desarrollo Tecnológico y Social (2020) 2 (2): 237-251 - Número especial COVID-19

colectivo - comunitarias para enfrentar la crisis sanitaria desde diálogos interculturales.

\section{Referencias bibliográficas}

CLACSO (2020). Informe: Los efectos socioeconómicos y culturales de la pandemia COVID-19 y del aislamiento social, preventivo y obligatorio en las comunidades indígenas de la RMBA, NOA, NEA y Patagonia. http://antropologia.institutos.filo.uba.ar/anuncio/informe-los-efectos-

socioecon\%C3\%B3micos-y-culturales-de-la-pandemia-covid-19-y-del-aislamiento-s

Colangelo, M.A. (2009). La salud infantil en contextos de diversidad sociocultural, en: Tamagno, Liliana (comp.) Pueblos indígenas. Interculturalidad, colonialidad, política. Buenos Aires: Biblos, 117-127.

de Sousa Santos, B. (2020). La cruel pedagogía del virus. Buenos Aires: CLACSO.

Filo-UBA y otros (2020). Informe ampliado: efectos socioeconómicos y culturales de la pandemia COVID-19 y del aislamiento social, preventivo y obligatorio en los Pueblos Indígenas en Argentina -Segunda etapa. http://antropologia.institutos.filo.uba.ar/sites/antropologia.institutos.filo.uba.ar/fil es/info covid 2daEtapa.pdf

LIAS (2017). Protocolo de Actuación para Organismos Gubernamentales de la Provincia de Buenos Aires que reciben demandas de personas, Comunidades y Pueblos Indígenas por la efectivización de sus derechos. Realizado por el 
Innovación y Desarrollo Tecnológico y Social (2020) 2 (2): 237-251 - Número especial COVID-19

Laboratorio de Investigaciones en Antropología Social (LIAS) de la Facultad de Ciencias Naturales y Museo de la Universidad Nacional de La Plata. https://www.defensorba.org.ar/pdfs/protocolo-pueblos-indigenas.pdf

Liliana Tamagno (2020) Presentación del Informe ampliado ante autoridades del INAI y de la Jefatura de Gabinete del Gobierno Nacional. 14 de agosto del 2020.

Maidana, C.; Ottenheimer, AC. y E. Rossi (2011) Comunidades indígenas y apropiación de TICs. Un nuevo espacio para la producción de conocimiento conjunto. En: De Souza, J. y C. Maidana (Comp.) Antropología de los nativos Estrategias sociales de los sujetos en la investigación. La Plata: EDULP.

Menéndez, E. (1972). Racismo, colonialismo y violencia científica. Revista Transformaciones, 47, 169-196.

Tamagno, L. (1991). La cuestión indígena en la Argentina y los censores de la indianidad. América Indígena, LI (1), 123-152.

Tamagno, L.; García, S. M.; Ibáñez Caselli, M. A.; García, M. C.; Maidana, C.; Alaniz, M. \& Solari Paz, V. (2005). Testigos y protagonistas: un proceso de construcción de conocimiento conjunto con vecinos Qom. Una forma de hacer investigación y extensión universitaria. Revista Argentina de Sociología, 3(5), 206-222. 